\title{
Cystic Chordoma of the Cavernous Sinus
}

\author{
Prashant SATHE, Amit MAHORE, Aadil CHAGLA \\ King Edward VII Memorial Hospital, Seth G.S. Medical College, Department of Neurosurgery, Mumbai, India
}

Corresponding author: Amit MAHORE amit2mahore@yahoo.co.in

\section{ABSTRACT}

Chordomas are the tumors commonly involving base of skull which are predominantly solid in consistency. A cystic chordoma of the cavernous sinus is an extremely rare entity and has never been reported. We report a 26-year-old male presenting with gradually progressive right-sided sixth nerve palsy and headache. Computed tomography and magnetic resonance imaging of the brain showed a cystic lesion in the right cavernous sinus. The patient was successfully treated using a transnasal endoscopic transsphenoidal approach. Histopathological examination of the lesion revealed chordoma. Our case is the first report of such a condition. Chordoma should be considered in the differential diagnosis of cystic lesions in the parasellar location. We briefly review the radiology, the pathology and management dilemmas of such lesions.

KEYWORDS: Cavernous sinus, Chordoma, Cyst

\section{INTRODUCTION}

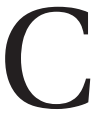
hordomas are tumors commonly involving the base of the skull, and are predominantly solid in consistency.

We report a 26 year-old-male patient who presented with a cystic lesion in right cavernous sinus that was confirmed as chordoma after histopathological examination. A cystic chordoma of the cavernous sinus is an extremely rare entity and has never been reported. The pathology and management dilemmas of such lesions have been briefly reviewed.

\section{- CASE REPORT}

A 26-year-old male patient presented with gradually progressive diplopia on right lateral gaze over 1 month period. It was accompanied by intermittent, mild, right hemi-cranial headache. On examination, the patient had lateral rectus palsy on the right side. Other cranial nerve examinations were within normal limits. Axial computed tomography (CT) scan of the brain showed a cystic lesion in the right parasellar region with erosion of the posterior clinoid process and lateral wall of the sphenoid sinus, and scalloping of the right petrous apex (Figure 1A). Magnetic resonance imaging (MRI) of the brain showed a well defined cystic lesion in the right cavernous sinus that was hypo-intense on T1-weighted images (Figure
1B) and hyper-intense on T2-weighted images (Figure 1C, D), with no enhancement on post-contrast images (Figure 1E) and without restriction of diffusion suggestive of an arachnoid cyst. It was displacing the right internal carotid artery anteroinferiorly in the cavernous course.

The patient was operated on using transnasal endoscopic transsphenoidal approach. The cystic tumor was removed. It contained grayish gelatinous liquid that was sent for histopathological examination. Postoperatively the patient had excellent improvement in his right sixth nerve palsy.

Histopathological examination showed small spindloid and epithelioid cells arranged in small clusters inside a myxoid stroma along with larger cells having a mutivacuolated cytoplasm and vesicular nuclei called physaliphorous cells, which is diagnostic of chordoma (Figure 2).

$\mathrm{MRI}$ of the brain was done at the follow-up of two years and showed a tiny residue. The patient was kept under regular follow-up (Figure 1F).

\section{DISCUSSION}

Chordomas arise from notochordal remnants along the craniospinal axis. Chordomas account for approximately 

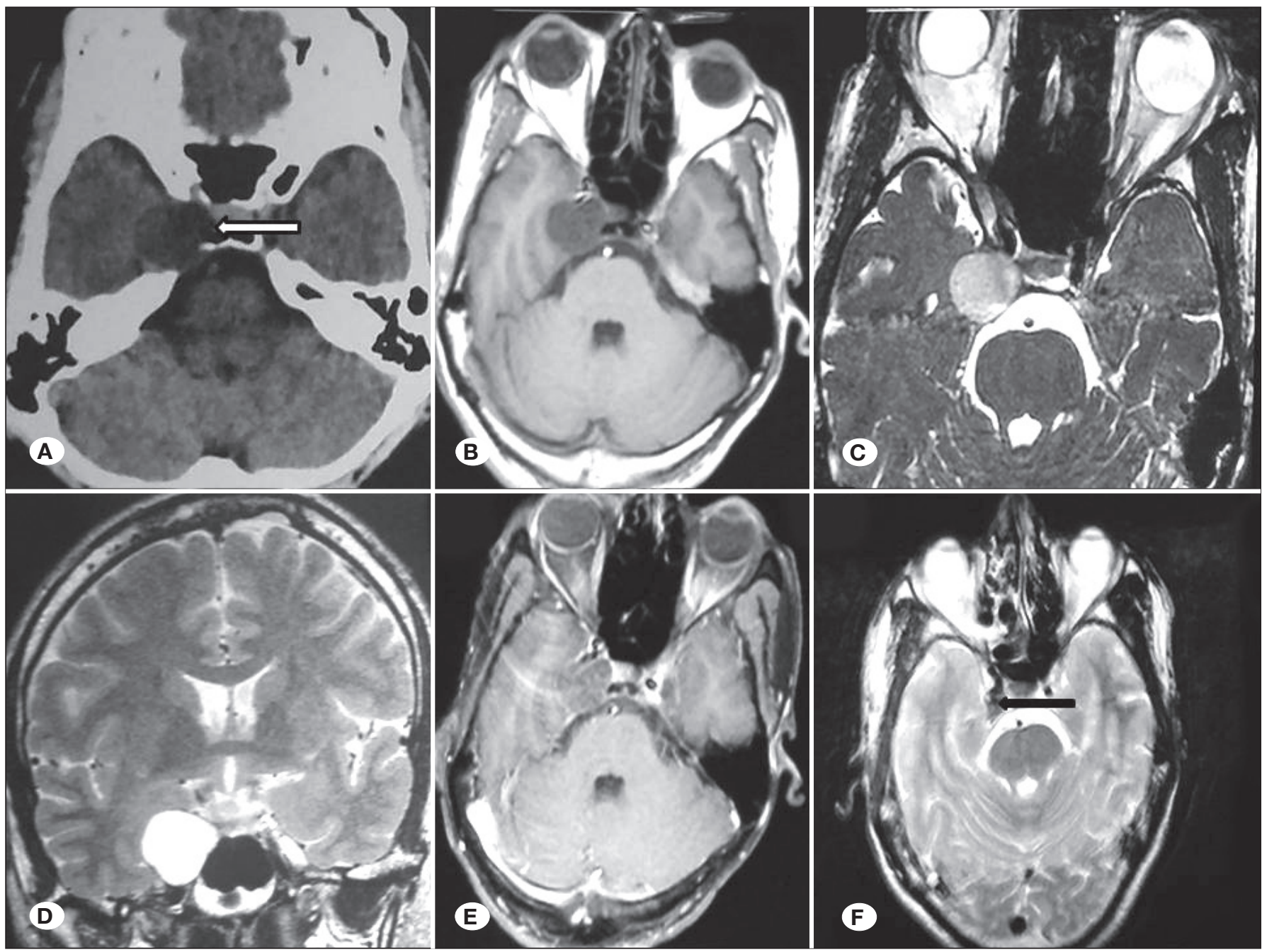

Figure 1: A) Axial image of CT scan showing cystic lesion in the right parasellar region with erosion of the posterior clinoid process and lateral wall of the sphenoid sinus and scalloping of the right petrous apex (white arrow); B) T1-weighted axial, C) T2-weighted axial and D) T2-weighted coronal MRI of the brain showing a cystic lesion of the right cavernous sinus. E) Post-contrast axial MRI showing the lesion with no contrast enhancement. F) Postoperative T2-weighted axial MRI showing almost complete decompression of the lesion with a tiny residue (black arrow).

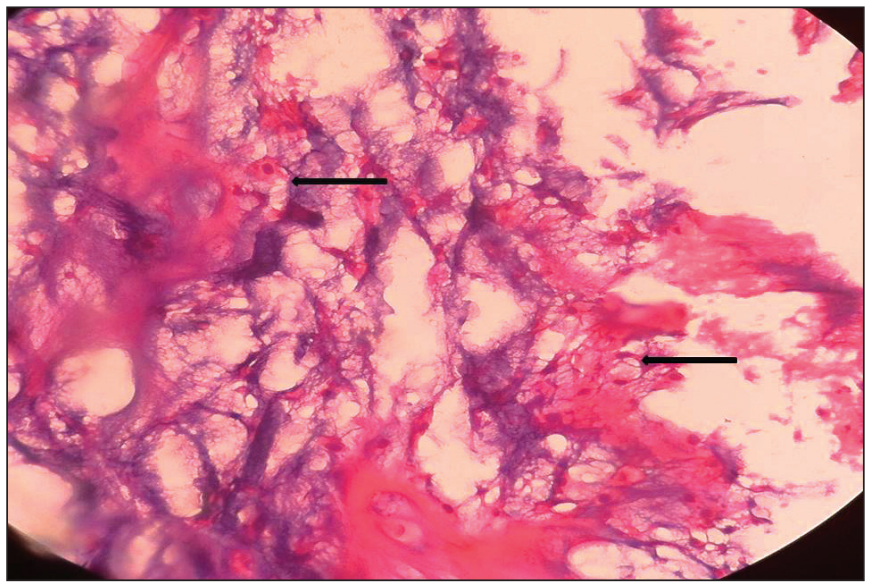

Figure 2: Histopathology image showing spindloid cells in myxoid stroma with physaliphorous cells (black arrows) (Hematoxylin\&Eosin, $x 40$ ).
$0.2 \%$ of all intracranial tumors and the most common site of origin is the clival region. The common age of presentation is the fourth and fifth decades $(2,4,5)$.

Histologically these tumors are benign in nature, but are classified as malignant because of the locally destructive nature, high rates of recurrence, and rare tendency to metastasize (11).

Pathologically, a chordoma is a solid, grayish, gelatinous tumor. Purely cystic chordomas are very rare and the exact percentage is not known. On histological examination, the mass shows spindloid and epithelioid tumor cells arranged in cords and clusters inside a myxoid stroma. The larger epithelioid cells with multivacuolated bubble like cytoplasm called physaliphorous cells are the characteristic cells of chordoma $(3,9)$. 
On MRI, chordoma appears as a solid mass lesion along the craniospinal axis in the midline which is hypo- to iso-intense on T1-weighted images and hyper-intense on T2-weighted images with heterogeneous contrast enhancement. CT scan shows heterogeneous contrast enhancement and bone erosion as well as destruction with accompanying calcifications $(6,7)$. The differential diagnosis includes chondrosarcoma, invasive pituitary macroadenoma or craniopharyngioma.

In this case, the diagnosis of arachnoid cyst was kept in mind considering the well defined cystic nature of the lesion which was hypo-intense on T1-weighted images and hyper-intense on T2-weighted images with no contrast enhancement and no diffusion restriction.

Radical tumor excision is the treatment of choice but safe and sufficient decompression is very important $(1,8)$. There are multiple approaches described for intracranial chordomas which include the transnasal endoscopic, extended subfrontal, subtemporal transmaxillary, transmandibular, orbitozygomatic, preauricular infratemporal and transcondylar approaches, but the appropriate approach is decided by the location and the extent of the tumor. The preferred approach for sellar-parasellar chordoma is the endoscopic endonasal approach. It provides excellent exposure to the sphenoid sinus, sellar-parasellar region, and upper and middle part of the clivus (10). Endoscopic assistance is helpful in enhancing lateral exposure. In this case, we preferred the transnasal endoscopic transsphenoidal approach as the lesion was totally inside the right cavernous sinus.

\section{CONCLUSION}

Cystic intracranial chordomas are very rare as compared to solid lesions. The differential diagnosis of cystic chordoma should be kept in mind in case of a cystic lesion in the sellarparasellar region.

\section{REFERENCES}

1. Al Mefty O, Borba LA: Skull base chordomas: A management challenge. J Neurosurg 86:182-189, 1997

2. Arnold H, Herrmann HD: Skull base chordoma with cavernous sinus involvement. Partial or radical tumour removal? Acta Neurochir (Wien) 83:31-37, 1987

3. Baratti D, Gronchi A, Pennacchioli E, Lozza L, Colecchia M, Fiore M: Chordoma: Natural history and results in 28 patients treated at a single institution. Ann Surg Oncol 10:291-296, 2003

4. Erdem E, Angtuaco EC, Van Hemert R, Park JS, AlMefty O: Comprehensive review of intracranial chordoma. Radiographics 23(4): 995-1009, 2003

5. Handa J, Suzuki F, Nioka H, Koyama T: Clivus chordoma in childhood. Surg Neurol 28:58-62, 1987

6. Leproux F, De Toffol B, Aesch B, Cotty P: MRI of cranial chordomas: The value of gadolinium. Neuroradiology 35: 543545, 1993

7. Nguyen RP, Salzman KL, Stambuk HE, Ahuja AT, Harnsberger HR: Extraosseous chordoma of the nasopharynx. Am J Neuroradiol 30:803-807, 2009

8. Raffel C, Wright DC, Gutin PH, Wilson CB: Cranial chordomas: Clinical presentation and results of operative and radiation therapy in twenty-six patients. Neurosurgery 17:703-710, 1985

9. Sell M, Sampaolo S, Di Lorio G, Theallier A: Chordomas: A histological and immunohistochemical study of cases with and without recurrent tumors. Clin Neuropathol 23(6):277285, 2014

10. Sen C, Triana AI, Berglind N, Godbold J, Shrivastava RK: Clival chordomas: Clinical management, results, and complications in 71 patients. J Neurosurg 113:1059-1071, 2010

11. Walcott BP, Nahed BV, Mohyeldin A, Coumans JV, Kahle KT, Ferreira MJ: Chordoma: Current concepts, management and future directions. Lancet Oncol 13:e69, 2012 